\title{
Strong Rashba-Type Spin Polarization of the Photocurrent from Bulk Continuum States: Experiment and Theory for Bi(111)
}

\author{
A. Kimura, ${ }^{1, *}$ E. E. Krasovskii, ${ }^{2,3,4}$ R. Nishimura, ${ }^{1}$ K. Miyamoto, ${ }^{5}$ T. Kadono, ${ }^{1}$ K. Kanomaru, ${ }^{1}$ E. V. Chulkov, ${ }^{2,3,6}$ \\ G. Bihlmayer, ${ }^{7}$ K. Shimada, ${ }^{5}$ H. Namatame, ${ }^{5}$ and M. Taniguchi ${ }^{1,5}$ \\ ${ }^{1}$ Graduate School of Science, Hiroshima University, 1-3-1 Kagamiyama, Higashi-Hiroshima 739-8526, Japan \\ ${ }^{2}$ Departamento de Física de Materiales, Facultad de Ciencias Químicas, Universidad del Pais Vasco/Euskal Herriko Unibertsitatea, \\ Apartado 1072, 20080 San Sebastián/Donostia, Basque Country, Spain \\ ${ }^{3}$ Donostia International Physics Center (DIPC), \\ Paseo Manuel de Lardizabal 4, 20018 San Sebastián/Donostia, Basque Country, Spain \\ ${ }^{4}$ IKERBASQUE, Basque Foundation for Science, 48011 Bilbao, Spain \\ ${ }^{5}$ Hiroshima Synchrotron Radiation Center, Hiroshima University, 2-313 Kagamiyama, Higashi-Hiroshima 739-0046, Japan \\ ${ }^{6}$ Centro de Física de Materiales CFM-Materials Physics Center MPC, Centro Mixto CSIC-UPV/EHU, Edificio Korta, \\ Avenida de Tolosa 72, 20018 San Sebastián, Spain \\ ${ }^{7}$ Institut für Festkörperforschung, Forschungszentrum Jülich, D-52425, Jülich, Germany
}

(Received 15 February 2010; published 13 August 2010)

\begin{abstract}
Strong spin polarization of the photocurrent from bulk continuum states of $\mathrm{Bi}(111)$ is experimentally observed. On the basis of ab initio one-step photoemission theory the effect is shown to originate from the strong polarization of the initial states at the surface and to be the result of the surface sensitivity of photoemission. Final state effects cause deviations of the $\mathbf{k}_{\|}$dependence of polarization from strictly antisymmetric relative to $\bar{\Gamma}$.
\end{abstract}

DOI: 10.1103/PhysRevLett.105.076804

PACS numbers: 73.20.At, 79.60.Bm

Spin-split energy-band structures induced by a broken space inversion symmetry with a strong spin-orbit coupling play an important role in spin injection, spin accumulation, and the generation of spin current, which would be key properties in the next generation of spintronics devices [13]. Rashba-type spin-orbit splitting of surface states on low index surfaces has been directly studied by angle-resolved photoemission spectroscopy (ARPES) for Au [4,5], $\mathrm{Bi}$ [6-9], Sb [10], and surface alloys [11-13]. The spin polarization of the surface states on $\mathrm{Au}(111)$ [5] and Bi(111) [14] was confirmed by spin-resolved (SR) ARPES. In general, the experimental effort in SRARPES on nonmagnetic solids has concentrated on low-dimensional systems [15]. Spin-orbit coupling is known to cause a spindependent photoemission also from bulk continuum states, and the cases hitherto observed are explained either by a final state effect (spin-dependent transmission of the photoelectron through the surface) $[16,17]$ or by special symmetry properties of initial states at a surface of reduced symmetry [18]. In the latter case, for $s$-polarized light or for nonpolarized light at non-normal incidence [19], the spin polarization of photoelectrons can occur in normal emission, which was experimentally verified in Refs. [20,21]. This is different from the Rashba effect, which is caused by the potential gradient in the surface perpendicular direction, and which occurs even at a surface of arbitrarily high symmetry but can be seen only in offnormal emission. In spite of its basic and general nature, such an effect in the continuum spectrum has not been reported yet.
This work presents the observation of a Rashba-type polarization of photocurrent from continuum spectrum states of $\mathrm{Bi}(111)$. The ARPES on $\mathrm{Bi}(111)$ was pioneered by Jezequel et al. [6] followed by the work by Tanaka et al. [7] with a higher energy resolution. Ast and Höchst resolved two surface states at the Fermi level [8] that were later identified as Rashba-split states by Koroteev et al. [9]. Both quantum-well and surface states were studied on thin films [22], and the spin character of the surface states at the Fermi energy was measured by SRARPES in Refs. [14,23].

The polarization of the bulk emission observed in the present work is similar to the Rashba effect for surface states: it is zero at normal emission $\mathbf{k}_{\|}=0$, and at $\mathbf{k}_{\|} \neq 0$ it may occur even at high-symmetry surfaces, in contrast to the Tamura-Piepke-Feder case [18]. Our theoretical analysis shows that the effect comes from the polarization of initial states at the surface due to the reflection of bulk Bloch states from the surface barrier, and the polarization of the photocurrent is the consequence of the surface sensitivity of photoemission.

Our SRARPES experiment on $\mathrm{Bi}(111)$ was performed on a clean surface obtained by repeated cycles of Ar-ion bombardment $(1.5 \mathrm{keV})$ and annealing at $450 \mathrm{~K}$ in ultrahigh vacuum. Cleanliness of the surface was confirmed by Auger electron spectroscopy and by a $1 \times 1$ low energy electron diffraction (LEED) pattern. SRARPES was measured using our homemade system equipped with the hemispherical electron analyzer connected to the retarding-type Mott spin polarimeter [24]. The sample temperature was set at $130 \mathrm{~K}$. The unpolarized He reso- 
nance line $(\hbar \omega=21.2 \mathrm{eV})$ was used. The angular acceptance of photoelectrons was set to $2^{\circ}$, and the estimated total energy resolution was $110 \mathrm{meV}$. The photon incidence angle was $50^{\circ}$ with respect to the center axis of the electron analyzer. The photoelectrons are collected along the $\bar{\Gamma} \bar{M}$ direction (i.e., $\mathbf{k}_{\|}$direction) of the hexagonal surface Brillouin zone (SBZ), see Ref. [7] for geometry.

Experimental energy distribution curves (EDCs) for two spin polarizations ( $\uparrow$ and $\downarrow$ ) perpendicular to the emission plane are shown in Figs. 1(a) and 1(b) for the emission angles $\theta$ from $-10^{\circ}$ to $+10^{\circ}$, and the data in a wider $k_{\|}$ range are summarized in Fig. 2.

Theoretically, the energy-momentum intensity distribution is obtained within an ab initio one-step theory of photoemission [25]. The occupied states are calculated for a 16 bilayer slab with a self-consistent full-potential [26] linear augmented plane wave method (LAPW). The two-component Koelling-Harmon approximation [27,28] for the relativistic Hamiltonian is employed. Final states are time-reversed LEED states taken in the scalar relativistic approximation. The inelastic scattering of outgoing electrons is included via an imaginary potential $V_{i}=1 \mathrm{eV}$, which is spatially constant in the crystal and equals zero in vacuum. The inelastic scattering rate is not strongly material dependent, and the value of $1 \mathrm{eV}$ is realistic for the present energy range, see Ref. [29]. The absorbing potential $V_{i}$ causes the LEED function to decay in space and is responsible for the surface sensitivity of the one-step simulation of photoemission. The method of calculation in the LAPW formalism was presented elsewhere [30]. Calculated spin-dependent energy-momentum intensity distributions are shown in Fig. 3. The light incidence is fixed at $50^{\circ}$ relative to the surface normal, which ensures that the asymmetry of the theoretical maps comes solely from the scattering of photoelectrons by the surface.
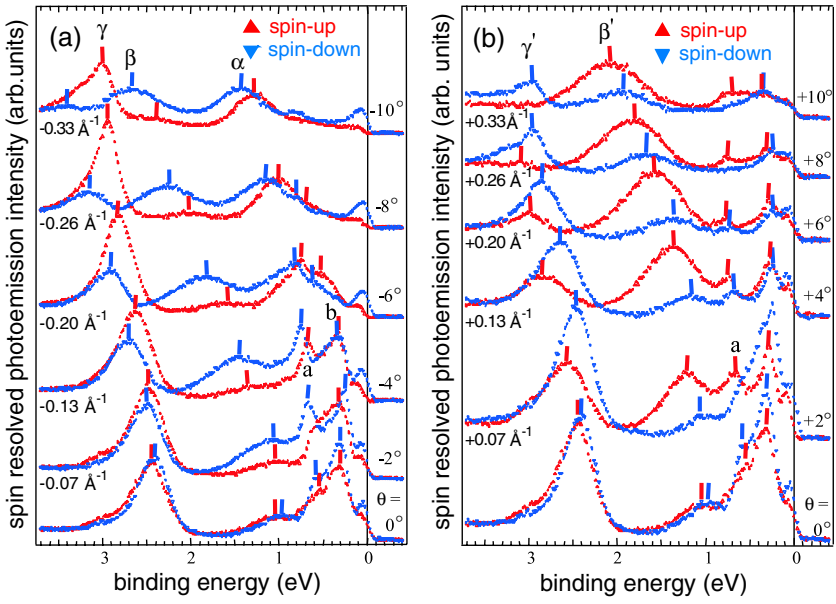

FIG. 1 (color online). Spin-resolved EDCs for Bi(111) surface from 0 to $3.7 \mathrm{eV}$ binding energy, (a) from $\theta=0^{\circ}$ to $-10^{\circ}$ and (b) from $\theta=0^{\circ}$ to $+10^{\circ}$. Corresponding wave numbers are presented for every EDC on the left side of each frame.
Our measured energies and $k_{\|}$dispersion of main spectral features agree well with previous results [7] and confirm the assignment in Ref. [7] of strong peaks at 0.3 and at $2.4 \mathrm{eV}$ in normal emission to a surface state and a surface resonance, respectively, and the shoulder at $0.6 \mathrm{eV}$ and broad hump at $1 \mathrm{eV}$ to bulk states. The surface state at $0.3 \mathrm{eV}$ comes out as negatively (spin- $\downarrow$ ) polarized; however, this surface state exists only in a narrow $k_{\|}$interval, so the observed polarization may be caused by the finite angular resolution in combination with the highly asymmetric intensity distribution, compare Figs. 2(c) and 2(d). More informative are the spectral features that are observed in a wide $k_{\|}$range.

The first new result of the present study is that the surface resonance at $2.4 \mathrm{eV}$ observed in Ref. [7] splits from the bulk continuum in going from $\bar{\Gamma}$ and becomes a surface state with a Rashba-type polarization-antisymmetric relative to the $\bar{\Gamma}$ point of the SBZ. The surface state disperses downwards from $2.4 \mathrm{eV}$ at $\bar{\Gamma}$, and its spin is positive for $-\theta$ (line $\gamma$ in Fig. 2) and negative for $+\theta$ (line $\gamma^{\prime}$ ). Its opposite-spin counterpart remains a surface resonance at all $k_{\|}$and follows the surface state at a larger binding energy. The peak intensity, however, shows a pronounced asymmetry: for positive $\theta$ the intensity rapidly decreases with $\theta$ for both spins, so the spin- $\uparrow$ structure almost disappears at $\theta=+10^{\circ}\left(k_{\|} \sim 0.33 \AA^{-1}\right)$. At negative $\theta$, on the contrary, the intensity first slightly increases, and beyond $-8^{\circ}\left(-0.26 \AA^{-1}\right)$ it decreases again. The calculated counterpart of $\gamma \gamma^{\prime}$ appears at $2.1 \mathrm{eV}$ at $\bar{\Gamma}$ and
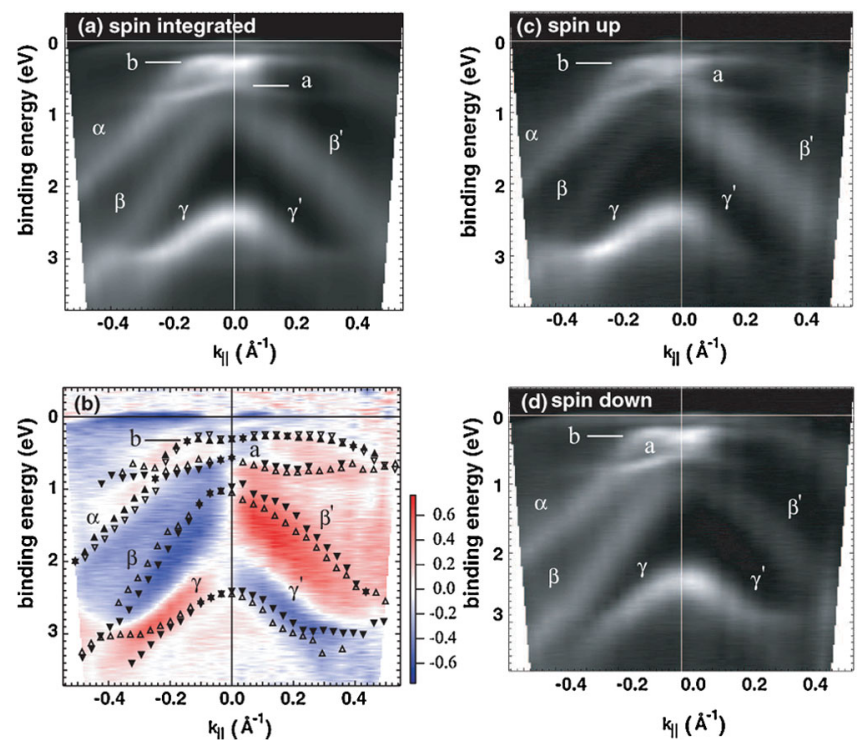

FIG. 2 (color). Experimental photoemission intensity distribution for $k_{\|}$in $\bar{\Gamma} \bar{M}$ direction. (a) spin-integrated intensity $I\left(E, k_{\|}\right)$. (b) spin polarization $P\left(E, k_{\|}\right)=\left(I_{\uparrow}-I_{\downarrow}\right) /\left(I_{\uparrow}+I_{\downarrow}\right)$ : positive (negative) polarization is plotted in red (blue); color strength gives the absolute value of $P\left(E, k_{\|}\right)$. (c) spin-up $I_{\uparrow}$ and (d) spindown $I_{\downarrow}$ intensities. Intensity maxima of spin- $\uparrow$ (spin- $\downarrow$ ) EDCs are plotted with open (filled) triangles in graph (b). 
(a) total intensity

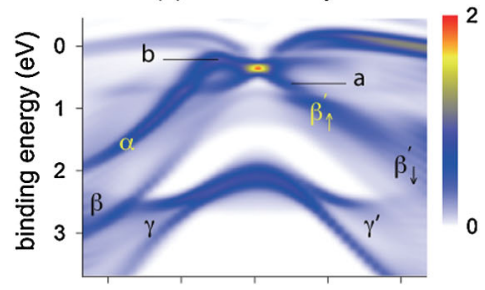

(b) spin polarization

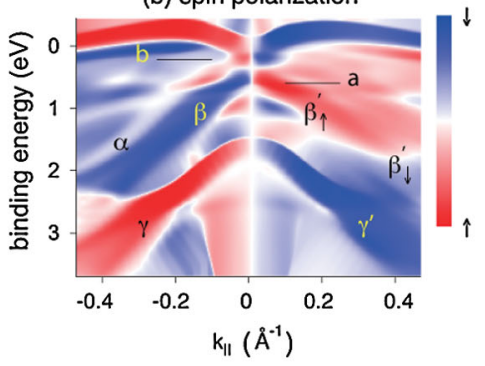

(c) intensity spin $\uparrow$

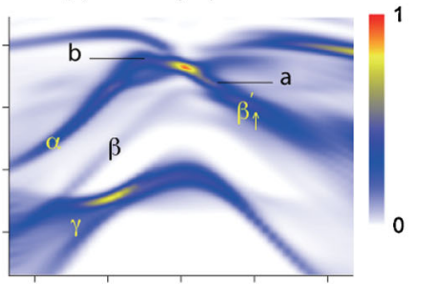

(d) intensity spin $\downarrow$

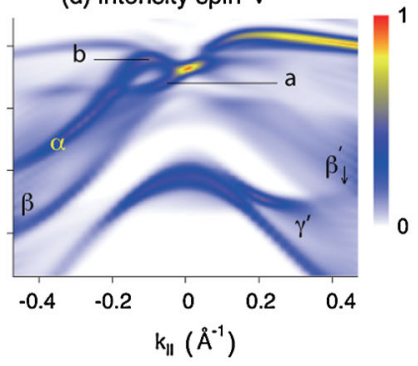

(e) total DOS

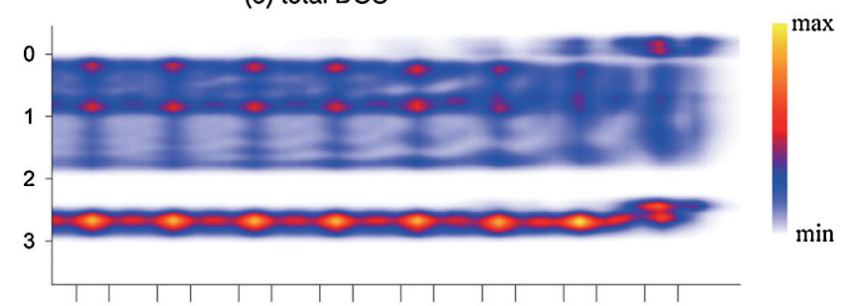

(f) spin density

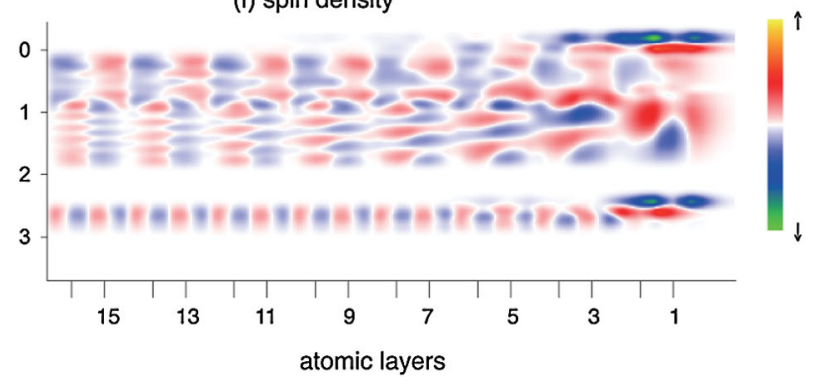

FIG. 3 (color). (a)-(d) Theoretical energy-momentum distribution of photoemission intensity for $\mathbf{k}_{\|}$along the $\bar{\Gamma} \bar{M}$ line of SBZ. (a) spin-integrated intensity, (b) spin polarization, (c) spin- $\uparrow$, and (d) spin- $\downarrow$ intensities. (e),(f) Local depth-resolved $k_{\|}$projected DOS $\rho_{\mathbf{k}_{\|}}(z, E)$ for $k_{\|}=0.2 \AA^{-1}$ : (e) total DOS (arb. units), (f) spin density $\rho_{\mathbf{k}_{\|}}^{\dagger}-\rho_{\mathbf{k}_{\|}}^{\downarrow}$. One-half of the slab is shown, vacuum is to the right. Tics in the horizontal axis show the positions of atomic layers.

merges at $\sim 0.35 \AA^{-1}$ with the bulk continuum in agreement with the experiment, see Figs. 2(a) and 3(a). The asymmetric spin-integrated intensity distribution $(\gamma$ is stronger than $\gamma^{\prime}$ ) is a vivid manifestation of the final state effect, which is correctly described by our theory.

Like for the surface states the polarization of the bulk derived states is roughly antisymmetric, but the asymmetry is much stronger. The shoulder at $0.6 \mathrm{eV}$ becomes a well-defined peak " $a$ " beyond $2^{\circ}$, see Fig. 1, which disperses from $0.7 \mathrm{eV}$ at $\theta= \pm 2^{\circ}$ to $0.8 \mathrm{eV}$ at $\pm 4^{\circ}$. A similar structure was observed in an ultrathin Bi film on $\operatorname{Si}(111)[14,22,23]$. Peak $a$ is strongly polarized: spin- $\downarrow$ dominates for negative $\theta$ and spin- $\uparrow$ for positive $\theta$. Both spin components closely follow each other, and at negative $\theta$ the line extends to larger $k_{\|}$than for positive $\theta$. Just above $a$, we resolve a concave down arc " $b$ ", which again is more intense at negative $\theta$ but its polarization is negligible. The lines $a$ and $b$ meet at $k_{\|} \sim 0.2 \AA^{-1}$, at which point an intense line $\alpha$ originates. The striking $k_{\|}$asymmetry of the intensity distribution of structures $a, b$, and especially $\alpha$ is well reproduced in our calculation, as well as the strong polarization of $a$ and weak of $b$ and $\alpha$, see Figs. 3(a)-3(d).

The weak structure at $E=1 \mathrm{eV}$ strongly enhances at off-normal emission. The maxima for both spins move downwards, with the slope $d E / d k_{\|}$being considerably steeper at negative $\theta$ (line $\beta$ ) than at positive $\theta$ (line $\beta^{\prime}$ ). [The intensity maxima of the EDCs are plotted in Fig. 2(b) with triangles.] Being unpolarized at $\theta=0$ it strongly polarizes already at small $\theta$, oppositely to the surface state $\gamma \gamma^{\prime}$ : for $-\theta$ the spin- $\downarrow$ peak dominates, and for $+\theta$ it is spin- $\uparrow$. However, both spin components are present for each $k_{\|}$direction, and the maxima are antisymmetrically shifted in energy: at positive $k_{\|}$the spin- $\uparrow$ peaks occur below the spin- $\downarrow$ peaks, and at negative $k_{\|}$they are above the spin- $\downarrow$ peaks. The theory well reproduces the polarization and the asymmetric dispersion of $\beta$ and $\beta^{\prime}$; however, it deviates from the experiment in smaller details: the measured $\beta_{\downarrow}^{\prime}$ line lies above $\beta_{\uparrow}^{\prime}$, whereas in the theory the former is slightly below the latter, see Figs. 3(c) and 3(d). One possible reason for the above discrepancies with the experiment is the deficiency of the two-component approximation for relativistic wave functions, which may cause errors of several tenths of an $\mathrm{eV}$ for the energies of $p$ states in crystals with strong spin-orbit interaction [28].

In order to understand the origin of the spin polarization of the photocurrent from bulk states we present in Figs. 3(e) and 3(f) the local depth-resolved $\mathbf{k}_{\|}$projected density of states (DOS) for $k_{\|}=0.2 \AA^{-1}$, which is defined as a sum of the densities of spin $\sigma$ [integrated over a surface parallel plane $z=$ const, with $\left.\mathbf{r}=\left(\mathbf{r}_{\|}, z\right)\right]$ over all states $\lambda$ with energy $E$ and Bloch vector $\mathbf{k}_{\|}$:

$$
\rho_{\mathbf{k}_{\|}}^{\sigma}(z, E)=\int d \mathbf{r}_{\|} \sum_{\lambda}\left|\psi_{\lambda \mathbf{k}_{\|}}^{\sigma}(\mathbf{r})\right|^{2} \delta\left(E_{\lambda \mathbf{k}_{\|}}-E\right) .
$$

For a true semi-infinite crystal $\Sigma$ means integration over the bulk continuum and summation over surface states. In our slab calculation, the $\mathbf{k}_{\|}$projected electronic structure is given by a discrete set of levels $\lambda$, and the $\delta$ function is replaced by a Gaussian of $0.15 \mathrm{eV}$ FWHM. The calculation is well converged with respect to the slab thickness: the six innermost bilayers come out practically identical and well represent the bulk electronic structure.

Three surface states are readily recognized by their large and strongly polarized DOS at the surface bilayer. Two of 
them are unoccupied at $k_{\|}=0.2 \AA^{-1}$, and the one at $2.4 \mathrm{eV}$ corresponds to the experimental spin- $\downarrow$ structure $\gamma^{\prime}$. Just below the surface state its opposite-spin counterpart is seen to be a surface resonance at the band maximum. The bulk continuum states are weaker polarized than the surface states, and, in contrast to the latter, their polarization density is very inhomogeneous. Deep in the bulk the $k_{\|}$resolved spin density changes sign on a unit cell scale, but the average over the unit cell is zero. In contrast, the net polarization over the two outermost bilayers is rather large, which gives rise to a spin-polarization of the photocurrent as a consequence of the surface sensitivity of photoemission. However, also in the surface layers the sign of the polarization changes with depth; in particular, note a curious resonance of the spin- $\downarrow$ density in the second bilayer at $1 \mathrm{eV}$ in Fig. 3(f). Thus, the polarization of photoelectrons can only roughly be inferred from the spin density of initial states.

The Rashba-type polarization at the surface can be understood as due to the beating between the bulk Bloch states incident on and reflected from the surface [31]. It leads to different density distributions for the two spineigenstates (in the Rashba model) of the semi-infinite crystal with the same $E$ and $\mathbf{k}_{\|}$. The Bloch eigenstates of the infinite crystal evidently have different wave functions for different spins, so they are reflected differently from the surface. The beating vanishes in going into the bulk, where the spin dependence of $\rho_{\mathbf{k}_{\|}}^{\sigma}(z, E)$ becomes much weaker and has the lattice periodicity.

To summarize, we have observed for the first time a strong spin polarization of bulk continuum states in photoemission from $\mathrm{Bi}(111)$ and explained it as coming from the surface sensitivity of the photoemission experiment. As in the Rashba model, the spin-resolved DOS is antisymmetric with respect to the $\bar{\Gamma}$ point. The spin polarization of the photocurrent roughly correlates with the polarization in the surface layers and is only approximately antisymmetric. The inequivalence of positive and negative $k_{\|}$leads to an asymmetry of the spin-integrated energy-momentum intensity distribution (both for surface and for bulk states) and to an asymmetry of the spin-polarization map for bulk states. These effects depend on the structure of final states (scattering of the photoelectron by the surface), and they are correctly reproduced within the one-step photoemission theory, which establishes their intrinsic surfacescattering origin.

In the present experiment the net polarization of the surface is zero. However, if the symmetry is broken by applying an electric field parallel to the surface the Fermi distribution is shifted relative to $\bar{\Gamma}$ and the surface may acquire a net magnetization, similarly to the magnetoelectric effect predicted for two-dimensional systems in Ref. [32]. Thus, the spin-orbit induced surface polarization appears to be a quite general phenomenon, which is not restricted to surfaces with surface states or quantum wells. This has far reaching implications, e.g., in the context of intrinsic spin torques that recently received attention in spintronic applications [33].

The SRARPES experiment was performed with the approval of the Proposal Assessing Committee of HSRC (Proposal No. 07-A-9). This work was financially supported by the JSPS Grant-in-Aid for Scientific Research (B) 20340092 .

*akiok@hiroshima-u.ac.jp

[1] Y. A. Bychkov and E. I. Rashba, JETP Lett. 39, 78 (1984).

[2] S. Datta and B. Das, Appl. Phys. Lett. 56, 665 (1990).

[3] Y. K. Kato, R. C. Myers, A.C. Cossard, and D. D. Awschalom, Science 306, 1910 (2004).

[4] S. LaShell, B. A. McDougall, and E. Jensen, Phys. Rev. Lett. 77, 3419 (1996).

[5] M. Hoesch et al., Phys. Rev. B 69, 241401(R) (2004).

[6] G. Jezequel, Y. Petroff, R. Pinchaux, and Félix Yndurain, Phys. Rev. B 33, 4352 (1986).

[7] A. Tanaka et al., Phys. Rev. B 59, 1786 (1999).

[8] C.R. Ast and H. Höchst, Phys. Rev. Lett. 87, 177602 (2001).

[9] Y. M. Koroteev et al., Phys. Rev. Lett. 93, 046403 (2004).

[10] K. Sugawara, T. Sato, S. Souma, T. Takahashi, M. Arai, and T. Sasaki, Phys. Rev. Lett. 96, 046411 (2006).

[11] C. R. Ast et al., Phys. Rev. Lett. 98, 186807 (2007).

[12] C. R. Ast et al., Phys. Rev. B 77, 081407(R) (2008).

[13] H. Bentmann et al., Europhys. Lett. 87, 37003 (2009).

[14] T. Hirahara et al., Phys. Rev. B 76, 153305 (2007).

[15] J. H. Dil, J. Phys. Condens. Matter 21, 403001 (2009).

[16] J. Kirschner, R. Feder, and J.F. Wendelken, Phys. Rev. Lett. 47, 614 (1981).

[17] H. P. Oepen, K. Hünlich, and J. Kirschner, Phys. Rev. Lett. 56, 496 (1986).

[18] E. Tamura, W. Piepke, and R. Feder, Phys. Rev. Lett. 59, 934 (1987).

[19] E. Tamura and R. Feder, Solid State Commun. 79, 989 (1991).

[20] B. Schmiedeskamp, B. Vogt, and U. Heinzmann, Phys. Rev. Lett. 60, 651 (1988).

[21] P. Stoppmanns et al., Phys. Rev. Lett. 66, 2645 (1991).

[22] T. Hirahara et al., Phys. Rev. Lett. 97, 146803 (2006).

[23] T. Hirahara et al., New J. Phys. 10, 083038 (2008).

[24] K. Iori et al., Rev. Sci. Instrum. 77, 013101 (2006).

[25] P. J. Feibelman and D. E. Eastman, Phys. Rev. B 10, 4932 (1974).

[26] E. E. Krasovskii, F. Starrost, and W. Schattke, Phys. Rev. B 59, 10504 (1999).

[27] D. D. Koelling and B. N. Harmon, J. Phys. C 10, 3107 (1977).

[28] A. H. MacDonald, W. E. Pickett, and D. D. Koelling, J. Phys. C 13, 2675 (1980).

[29] E. E. Krasovskii et al., Phys. Rev. Lett. 98, 217604 (2007); E. E. Krasovskii et al., Phys. Rev. B 78, 165406 (2008).

[30] E. E. Krasovskii and W. Schattke, Phys. Rev. B 59, R15 609 (1999).

[31] E. E. Krasovskii et al., Phys. Rev. B 75, 045432 (2007).

[32] V. M. Edelstein, Solid State Commun. 73, 233 (1990).

[33] I. M. Miron et al., Nature Mater. 9, 230 (2010). 